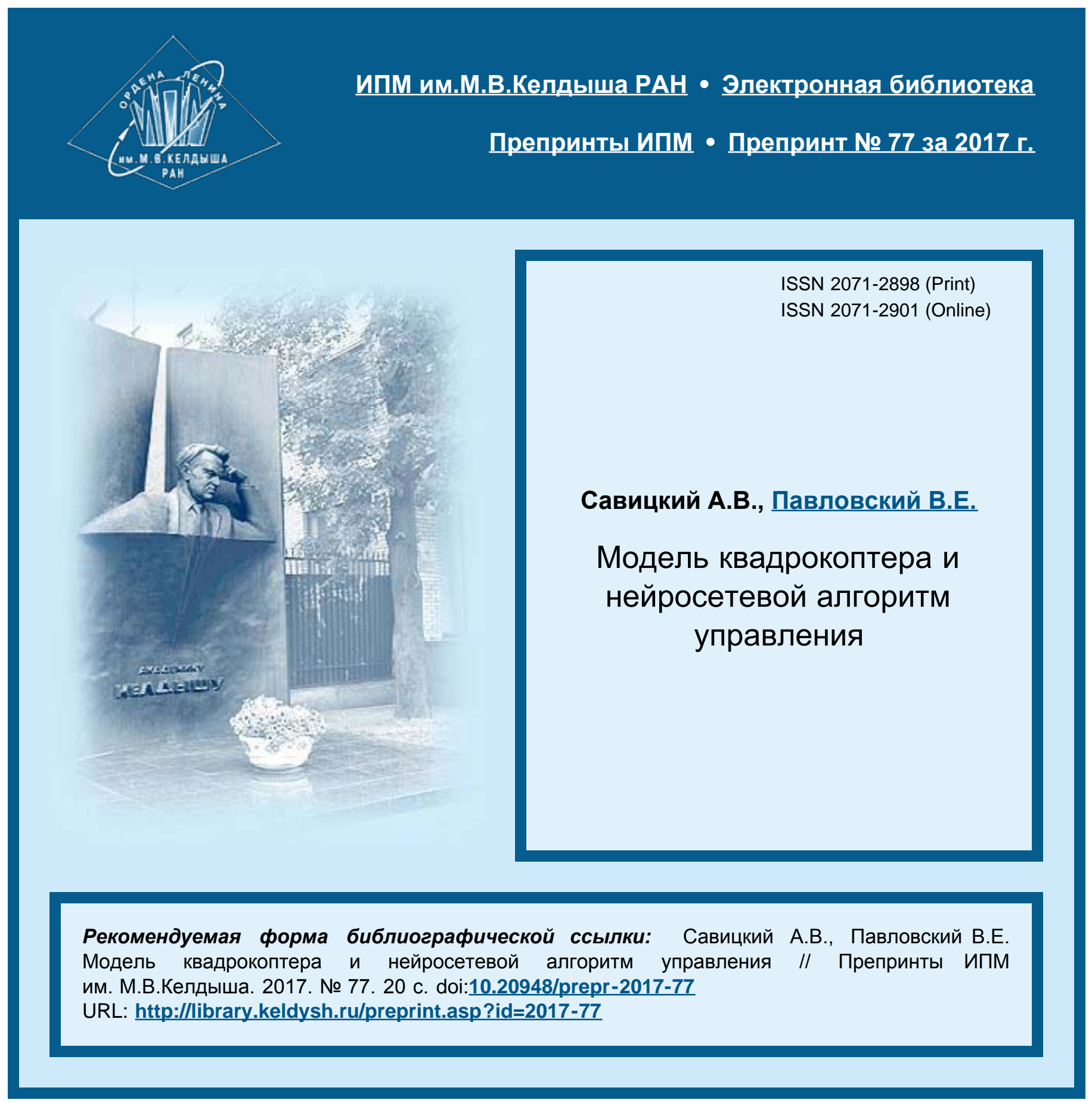




\author{
О рд н н а Л н и на \\ ИНСТИТУТ ПРИКЛАДНОЙ МАТЕМАТИКИ \\ имени М.В. Келдыша \\ Р ос ий ск ой а кадеми и наук
}

\begin{abstract}
А.В.Савицкий, В.Е.Павловский
МОДЕЛЬ КВАДРОКОПТЕРА

И НЕЙРОСЕТЕВОЙ АЛГОРИТМ УПРАВЛЕНИЯ
\end{abstract}

Москва - 2017 


\section{УДК 623.746-519}

\section{Савицкий А.В., Павловский В.Е}

\section{Модель квадрокоптера и нейросетевой алгоритм управления}

В работе представлена теоретико-механическая модель беспилотного летательного аппарата - квадрокоптера. Изучены такие базовые траектории, как взлет-парениепосадка, полет по прямой. Рассмотрен нейросетевой алгоритм построения управляющих воздействий для базовых траекторий. На основе результатов численного моделирования построена обучающая выборка, построена сходящаяся нейронная сеть. Показано, что построенный алгоритм управления удовлетворительно работает для случая базовых траекторий. Также представлены результаты работы нейросетевого алгоритма при наличии погрешности позиционного датчика.

Ключевые слова: квадрокоптер, нейронная сеть, алгоритм управления, нейросетевой регулятор.

\section{Alexander V. Savitskiy, Vladimir E. Pavlovsky Model of quadrotor and neural network algorithm of vehicle control}

This paper presents a theoretical mechanical model of an unmanned aerial vehicle quadrocopter. It studies such basic trajectories as take-off, landing, hovering, flying in a straight line. This work also considers convergent neural network algorithm of control. Based on the results of numerical simulation a training set and a neural network were constructed. It is shown that the constructed control algorithm works satisfactorily in the case of basic trajectories. Besides this work presents results for the case when the position sensor has an error.

Key words: quadrocopter, neural network control algorithm, neural network controller. 


\section{Введение}

В течение последних лет тема беспилотных летательных аппаратов (БПЛА) получает все большее распространение. Особый интерес вызывают мультикоптерные роботы - устройства, приводимые в движение $n$ роторами (как правило, их количество - от 1 до 8). Случай одного ротора - это вертолет, четырех - квадрокоптер (коптер) - основной объект изучения данной работы [1].

Использование стандартных датчиков позиционирования (акселерометр, гироскоп) требует специальных исследований в связи с тем, что накапливаемая ошибка может значительно исказить выходную информацию. Поэтому большая часть всех публикаций посвящена соответствующим алгоритмам построения управлений и сравнению их эффективности, в том числе с использованием указанных датчиков. Среди этих работ можно выделить несколько групп:

1) метод построения управления, основанный на теории Ляпунова, позволяющие в определенной постановке достичь асимптотической устойчивости летательного аппарата [2];

2) алгоритм управления, в основе которого лежит пропорциональноинтегрально-дифференциальный (ПИД) регулятор; его основное преимущество заключается в экспоненциальной сходимости, упрощенной реализации, так как она не требует специфических параметров модели [3];

3) энергетические методы, применимые для пассивных систем с недостатком управляющих воздействий [4];

4) методы, основанные на визуальном управлении от видеокамеры (видеокамер), часто используются на взлете-посадке;

5) методы, основанные на управлении с помощью нейросетевого регулятора, используемого в задачах стабилизации, при поиске оптимальных параметров регулятора [5].

В отдельный класс задач можно выделить исследование проблем предотвращения столкновений для нескольких роботизированных систем в группе [10].

Особое место занимают методы, основанные на нейронных сетях. Задачи, в которых применяются нейросетевые контроллеры, можно разделить на два класса: построение управления для определенных режимов полета и отдельных траекторий и задачи стабилизации по всем или по части переменных [11].

Интересные результаты получены командой американских ученых из Университета Миссури [12], разработавших алгоритм управления группой квадрокоптеров. Данный алгоритм содержит два двуслойных нейросетевых контроллера. Первый используется для синтеза управляющих воздействий ведущего коптера, второй используется для стабилизации группового полета, он работает на основе данных с беспроводных бортовых датчиков. Последний контроллер в качестве входных параметров получает состояния системы, а на 
выходе выдает оптимальное управление для движения с минимальным отклонением от траектории ведущего коптера. Описанные в работе контроллеры позволяют учитывать аэродинамические эффекты и внешние возмущения. Также в этой работе представлен метод оптимизации каналов связи между квадрокоптерами, использующий теорию графов.

Есть работы по применению нейросетевого метода в изучении динамики вертолета. Например, в работе из Политехнического университета Мадрида [13] представлен гибридный контроллер, состоящий из двух нейронных сетей: Джордана и Элмана (т.е. рекуррентных сетей с обратными связями). В работе показано, что оптимальное управление коптером находится при отдельном рассмотрении различных этапов полета, в работе не строится общее оптимальное управление для всего полета.

Использование нейросетевого контроллера для управления высотой полета представлено в тезисах конференции [14]. В них приведено описание совместной работы пропорционально-интегрально-дифференциального и нейросетевого регуляторов. Одной из основных особенностей полученного алгоритма является быстрая адаптация к внешним воздействиям.

Целью настоящей работы является моделирование динамики квадрокоптера, изучение базовых траекторий, построение нейроалгоритмов управления для базовых траекторий и изучение влияния погрешностей системы на действие нейросетевого контроллера для задач взлета-посадки-парения. Одно из назначений этого контроллера - преодоление проблемы дефицита управлений, т.к. квадрокоптер - это система с их недостатком: система имеет (в полете) 6 степеней свободы и только 4 управления. Другая цель заключается в возможности реализации быстрых вычислений, т.к. нейросетевой метод относится к параллельным методам, работающим за малое число тактов вычислений.

Выбор нейросетевого метода связан, в первую очередь, с тем, что при реальном полете мультироторного робота появляется большое количество эффектов и воздействий, которые иначе трудно математически смоделировать и парировать. Например, при быстром полете воздушный поток может вызывать осевые вибрации вращающегося винта, называемые в литературе "blade flapping". Это связано с тем, что относительная скорость воздуха для "набегающей" лопасти винта сильно отличается от скорости воздуха для противоположной - "уходящей" - лопасти. Это приводит к тому, что подъемная сила каждой лопасти в течение одного оборота может меняться, вызывая тем самым колебания оси винта и соответственно воздействуя на летящий аппарат. Но в данной работе рассмотрены только умеренные маневры, поэтому этот эффект не будет учтен.

Также существует актуальная задача корректировки полета при появлении внешних возмущений, например ветра. С этой задачей связано 2 типа проблем. Первая - ветер сложно измерить, а получить информацию о его воздействии можно с навигационных бортовых датчиков, которые имеют определенную погрешность и шум. Вторая проблема связана с задержкой по времени между 
обнаружением отклонения и корректировкой полета. Поэтому также имеется задача оптимизации вычислительной схемы для повышения ее быстродействия.

\section{1. Описание модели}

Настоящая работа дополняет исследования, выполненные в [16], в которых также использовалась полная модель квадрокоптера, но она была дана без вывода. Изложение в настоящей работе начнем с полного вывода уравнений модели квадрокоптера. Этот раздел можно рассматривать и как дополнение к [16].

В качестве модели квадрокоптера рассмотрим плоское тело, состоящее из корпуса и 4 пропеллеров (рис.1). Корпусом будем считать 2 одинаковых стержня длины $2 l$, пересекающиеся в точке $A$ под прямым углом. Масса каждого стержня равна $m_{0} / 2$.

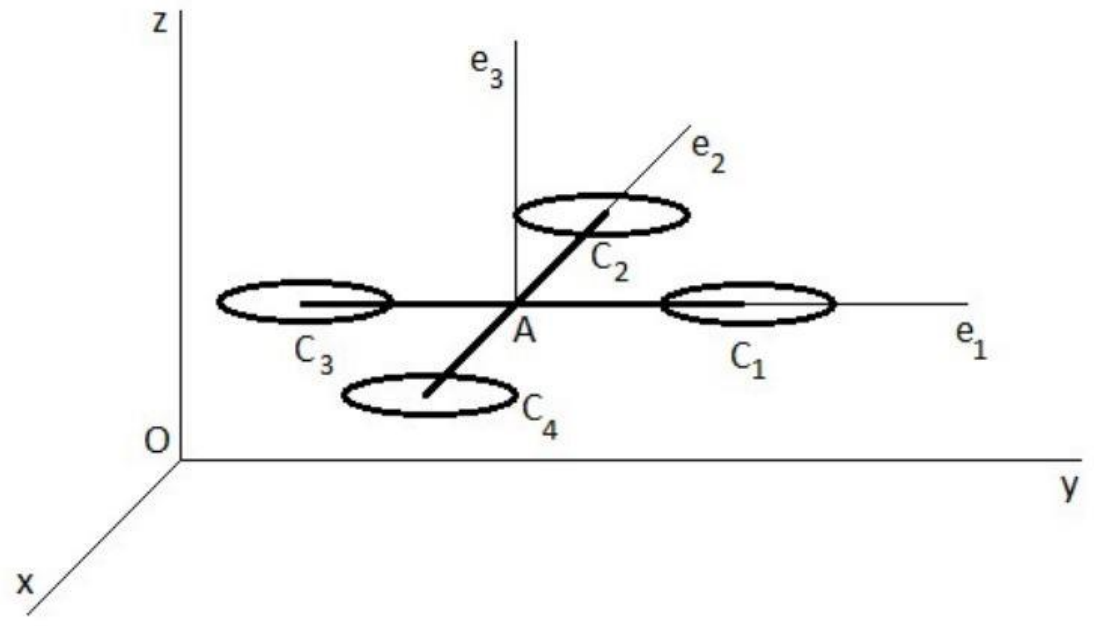

Рис. 1. Концептуальная модель квадрокоптера

Предполагается, что пропеллерами являются диски, закрепленные на концах стержней в точках $C_{1}, C_{2}, C_{3}, C_{4}$. Диски имеют радиус $r$, массу $m_{i}$. Центр масс робота $M=m_{0}+4 m_{i}$ находится в точке $A$.

Пусть $\{O x y z\}$ - правая инерциальная система координат, причем $O z$ направленна вертикально вверх. Положение центра масс тела определяется вектором $\xi=(x, y, z)$.

Пусть $\left\{\mathrm{Ae}_{1} e_{2} e_{3}\right\}$ - правая система координат, жестко связанная с роботом. Выберем ось $O e_{1}$ по направлению "руки" летательного аппарата, соответствующейэ точке $C_{1}$, ось $O e_{2}-$ перпендикулярно $O e_{1}$ в плоскости робота.

\section{1. Основные предположения}

1) При вычислении кинетического момента и других динамических характеристик пропеллера будем предполагать, что его тензор инерции относительно точки крепления имеет такой же вид, как у однородного диска, который назовем диском несущего винта. 
2) Будем рассматривать только умеренные маневры. Соответственно, мы пренебрегаем упругостью лопастей несущего винта, и диск несущего винта считается бесконечно жестким и бесконечно тонким. Диск все время вращается в плоскости, перпендикулярной оси $O e_{3}$.

3) Подъемная сила каждого диска создает силу тяги $u_{i}$, приложенную в точке $C_{i}$ и все время направленную вдоль оси $O e_{3}$.

4) Управление ориентацией задается с помощью трех независимых вращающих моментов $\left\{\Gamma_{1}, \Gamma_{2}, \Gamma_{3}\right\}$ вокруг каждой из трех осей системы координат $\left\{\mathrm{Ae}_{1} e_{2} e_{3}\right\}$. Эти вращающие моменты приложены непосредственно к корпусу и не порождают никаких сил поступательного движения, связанных со вторичными аэродинамическими эффектами или упругостью лопастей несущих винтов.

5) Полагая, что линейная скорость квадрокоптера и его вращения существенно меньше скорости вращения винтов, будем считать, что воздух оказывает сопротивление только на лопасти несущих винтов, создавая тем самым отрицательные вращательные моменты.

6) Векторы угловых скоростей винтов, расположенных в точках $C_{i}$ для нечетного $i$, сонаправлены вектору $e_{3}$, остальные - противонаправлены.

\section{2. Вычисление лагранжиана}

Ориентация корпуса робота задается с помощью трех углов Крылова $v=(\varphi$, $\psi, \theta)$. Соответствующую матрицу перехода в неподвижной системе координат обозначим $R$. Кроме того, чтобы учесть динамику несущих винтов, введем углы поворота лопастей $\dot{\gamma}_{1}, \dot{\gamma}_{2}, \dot{\gamma}_{3}$, и $\dot{\gamma}_{4}$. От них будут зависеть управляющие моменты.

Таким образом, обобщенные координаты для робота имеют вид: $q=(x, y, z$, $\varphi, \psi, \theta)$.

Обозначим $I_{A}$ момент инерции корпуса относительно точки $A$, выраженный в подвижной системе координат; $\Omega_{\mathrm{A}}$ - угловая скорость корпуса в той же системе координат.

Кинетическая энергия поступательного движения робота задается по формуле:

$$
T_{\text {trans }}=\frac{M}{2}(\dot{\xi}, \dot{\xi}) .
$$

Кинетическая энергия вращения корпуса:

$$
T_{\text {rotA }}=\frac{1}{2}\left(\Omega_{A}, \mathrm{I}_{A} \Omega_{A}\right),
$$

где $I_{A}=\operatorname{diag}\left(\mathrm{I}_{1}^{A}, \mathrm{I}_{2}^{A}, \mathrm{I}_{3}^{A}\right)$, причем в силу симметрии $\mathrm{I}_{1}^{A}=\mathrm{I}_{2}^{A}$.

В силу Предположения 2 угловая скорость лопастей в подвижной системе координат выражается по формуле:

$$
\Omega_{C_{i}}=\Omega_{A}+(-1)^{i+1} \dot{\gamma}_{i} e_{3} .
$$


Предположим, что несущие винты в процессе собственного вращения образуют диск с центром масс в точке $C_{i}$ и диагональным тензором инерции $I_{C}$ в подвижной системе координат: $I_{C}=\operatorname{diag}\left(\mathrm{I}_{1}^{C}, \mathrm{I}_{2}^{C}, \mathrm{I}_{3}^{C}\right)$, причем в силу симметрии $\mathrm{I}_{1}^{A}=\mathrm{I}_{2}^{A}$.

Таким образом, суммарная кинетическая энергия вращения квадрокоптера выглядит следующим образом:

$$
\begin{aligned}
& T_{\text {rotA }}=\frac{1}{2}\left(\Omega_{A}, \mathrm{I}_{A} \Omega_{A}\right)+\frac{1}{2} \sum_{i=1}^{4}\left(\Omega_{C_{i}}, \mathrm{I}_{C} \Omega_{C_{i}}\right)= \\
& =\frac{1}{2}\left(\Omega_{A},\left(\mathrm{I}_{A}+4 \mathrm{I}_{C}\right) \Omega_{A}\right)+\frac{1}{2} \mathrm{I}_{3}^{c}\left(\dot{\gamma}_{1}^{2}+\dot{\gamma}_{2}^{2}+\dot{\gamma}_{3}^{2}+\dot{\gamma}_{4}^{2}\right)+ \\
& +\left(\dot{\gamma}_{1}-\dot{\gamma}_{2}+\dot{\gamma}_{3}-\dot{\gamma}_{4}\right)\left(\Omega_{A} ; \mathrm{I}_{C} e_{3}\right)
\end{aligned}
$$

Единственный потенциал, который будет учтен, - потенциал силы тяжести: $V=m g z$.

Угловая скорость в подвижной системе координат имеет следующий вид:

$$
\Omega_{A}=\left(\begin{array}{c}
\dot{\theta}-\dot{\varphi} \sin \psi \\
\dot{\psi} \cos \theta+\dot{\varphi} \cos \psi \sin \theta \\
\dot{\varphi} \cos \psi \cos \theta-\dot{\psi} \sin \theta
\end{array}\right) .
$$

Положим по определению:

$$
W_{v}:=\left(\begin{array}{ccc}
-\sin \psi & 0 & 1 \\
\cos \psi \sin \theta & \cos \theta & 0 \\
\cos \psi \cos \theta & -\sin \theta & 0
\end{array}\right) .
$$

Тогда

$$
\dot{v}:=\left(\begin{array}{c}
\dot{\varphi} \\
\dot{\psi} \\
\dot{\theta}
\end{array}\right)=W_{v}^{-1}\left(\begin{array}{c}
\dot{\theta}-\dot{\varphi} \sin \psi \\
\dot{\psi} \cos \theta+\dot{\varphi} \cos \psi \sin \theta \\
\dot{\varphi} \cos \psi \cos \theta-\dot{\psi} \sin \theta
\end{array}\right) .
$$

Заметим, что $\operatorname{det} W_{v}=-\cos \psi$, то есть кинематическое преобразование будет невырожденным для всех ориентаций, за исключением тех, у которых $\psi=\pi / 2+$ $+\pi k, k$ - целое.

Полная функция Лагранжа имеет вид:

$$
L(q, \dot{q})=T_{\text {trans }}+T_{\text {rot }}-V .
$$

\section{3. Обобщенные силы}

В соответствии с Предположением 3 существуют 4 силы поступательного движения и все они направлены вдоль оси $e_{3}$. Следовательно, направление силы поступательного движения, приложенной к корпусу, определяется его 
ориентацией. В инерциальной системе координат направление силы тяги задается вектором: $G(\eta)=R \mathrm{e}_{3}$.

Поэтому суммарную силу тяги, рассматриваемую в качестве управления, можно выразить в инерциальной системе отсчета следующим образом:

$$
F_{\xi}=\left(u_{1}+u_{2}+u_{3}+u_{4}\right) R \mathrm{e}_{3}=\left(\begin{array}{c}
\left(u_{1}+u_{2}+u_{3}+u_{4}\right)(\cos \varphi \sin \psi \cos \theta+\sin \varphi \sin \theta) \\
\left(u_{1}+u_{2}+u_{3}+u_{4}\right)(\sin \varphi \sin \psi \cos \theta-\cos \varphi \sin \theta) \\
\left(u_{1}+u_{2}+u_{3}+u_{4}\right) \cos \varphi \cos \theta
\end{array}\right) .
$$

(9)

Три вращательных момента из Предположения 4, являющиеся управлениями, имеют следующие выражения в подвижной системе координат:

$$
\begin{aligned}
& \Gamma_{1}=\left(u_{2}-u_{4}\right) l e_{1}, \\
& \Gamma_{2}=\left(u_{3}-u_{1}\right) l e_{2}, \\
& \Gamma_{3}=\left(-M_{1}+M_{2}-M_{3}+M_{4}\right) e_{3}=M_{0} e_{3,}
\end{aligned}
$$

где $M_{i}$ - момент сопротивления $i$-го винта.

В итоге, обобщенные силы, зависящие от переменных $v$, имеют вид

$$
\tau^{0}:=\left(\begin{array}{l}
\tau_{\varphi} \\
\tau_{\psi} \\
\tau_{\theta}
\end{array}\right)=W_{v}^{-1}\left(\begin{array}{l}
\Gamma_{1} \\
\Gamma_{2} \\
\Gamma_{3}
\end{array}\right)=\left(\begin{array}{l}
\frac{\sin \theta}{\cos \psi}\left(u_{3}-u_{1}\right) l+\frac{\cos \theta}{\cos \psi} M_{0} \\
\cos \theta\left(u_{3}-u_{1}\right) l+\sin \theta M_{0} \\
\left(u_{2}-u_{4}\right) l+\operatorname{tg} \psi\left(\sin \theta l\left(u_{3}-u_{1}\right)+\cos \theta M_{0}\right)
\end{array}\right)
$$

\section{4. Модель подъемной силы и момента несущего винта}

В основу составления модели подъемной силы и момента несущего винта была положена статья [1], в которой изучен вертолет, установленный на экспериментальной платформе. Рассматривая малый элемент лопасти, имеем следующие выражения для аэродинамической силы и момента:

$$
\begin{aligned}
& u_{i}=\frac{\rho h c a V_{i} r^{2}}{4} \dot{\gamma}_{i}, \\
& M_{i}=\frac{\rho h c a r^{4} c_{d} \dot{\gamma}_{i}^{2}}{8},
\end{aligned}
$$

$\rho$ - плотность воздуха, $h$ - количество винтов, $c$ - ширина лопасти, $a-$ угловой коэффициент кривой подъема, $r$ - радиус диска винта, $V_{i}$ - индуцированная скорость парения, $c_{d}-$ коэффициент сопротивления. 
Введем дополнительные обозначения:

$$
k_{1}=\frac{\rho h c a V_{i} r^{2}}{4}, k_{2}=\frac{\rho h c c_{d} r^{4}}{8},
$$

тогда:

$$
u_{i}=(-1)^{i+1} k_{1} \dot{\gamma}_{i} \text { и } M_{i}=(-1)^{i} k_{2} \dot{\gamma}_{i}^{2} .
$$

\section{5. Уравнения Лагранжа}

Для начала введем обозначения: $A=I_{1}^{a}+4 I_{1}^{c}, \mathrm{D}=I_{3}^{a}+4 I_{3}^{c}$.

Уравнения Лагранжа:

$$
\frac{d}{d t} \frac{\partial L}{\partial \dot{q}}-\frac{\partial L}{\partial q}=Q
$$

где $Q$ - обобщенные силы,

$L(q, \dot{q})=T_{\text {trans }}+T_{\text {rot }}-U=$

$=\frac{1}{2} A\left(\dot{\theta}^{2}+\dot{\varphi}^{2} \sin ^{2} \psi-2 \dot{\varphi} \dot{\theta} \sin \psi+\dot{\psi}^{2} \cos ^{2} \theta+\dot{\varphi}^{2} \cos ^{2} \psi \sin ^{2} \theta+2 \dot{\varphi} \dot{\psi} \cos \theta \sin \theta \cos \psi\right)+$

$+\frac{1}{2} D\left(\dot{\psi}^{2} \sin ^{2} \theta+\dot{\varphi}^{2} \cos ^{2} \psi \cos ^{2} \theta-2 \dot{\varphi} \dot{\psi} \cos \theta \sin \theta \cos \psi\right)+\frac{1}{2} \mathrm{I}_{2}^{c}\left(\dot{\gamma}_{1}^{2}+\dot{\gamma}_{2}^{2}+\dot{\gamma}_{3}^{2}+\dot{\gamma}_{4}^{2}\right)$

$-\mathrm{I}_{3}^{c}\left(\dot{\gamma}_{1}-\dot{\gamma}_{2}+\dot{\gamma}_{3}-\dot{\gamma}_{4}\right)(\dot{\varphi} \cos \psi \cos \theta-\dot{\psi} \sin \theta)$

Исходя из построенной модели, получаем уравнения Лагранжа:

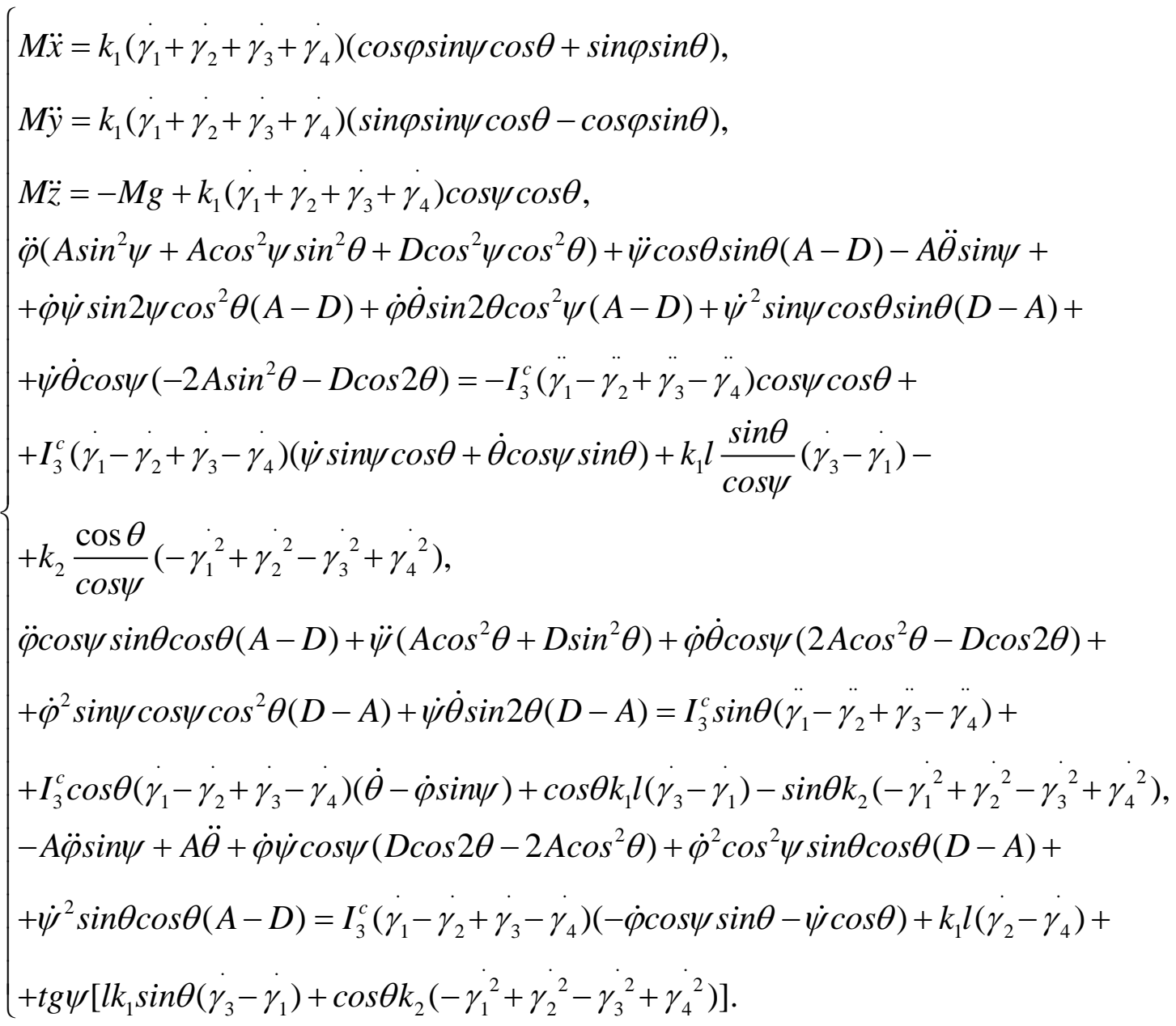


Полученную систему шести дифференциальных уравнений второго порядка можно представить в виде $K \ddot{q}=N$, где $K$ - симметричная матрица с определителем $A^{2} D \cos ^{2} \psi, N-$ правая часть уравнений. Стоит отметить, что определитель матрицы $K$ не равен нулю, так как мы рассматриваем случай $\psi \neq$ $\pi / 2+\pi k, k$ - целое. Следовательно, можем вычислить обратную матрицу $K^{-1}$. Далее это будет использовано для приведения полученной системы дифференциальных уравнений к виду Коши и численного интегрирования.

\section{2. Моделирование и алгоритмы управления}

Ввиду громоздкости полученных уравнений динамики представляется сложным найти аналитическое решение в общем виде. В связи с этим часть исследований опирается на результаты численного моделирования.

Численное интегрирование полученной системы уравнений осуществлялось с помощью программы MATLAB R2013а методом Рунге-Кутты. Был рассмотрен квадрокоптер со следующими параметрами:

$$
\begin{aligned}
& M=1 \mathrm{\kappa}, \\
& m_{i}=0,1 \kappa \Gamma, \\
& l=0,4 \mathrm{M}, \\
& p=4, \\
& r=0,05 \mathrm{M}, \\
& c=0,01 \mathrm{M}, \\
& \rho=1,3 \kappa \Gamma / \mathrm{M}^{3}, \\
& V_{i}=2 \mathrm{M} / \mathrm{c}, \\
& c_{d}=50 .
\end{aligned}
$$

\section{1. Взлёт-посадка}

В качестве одной из базовых траекторий рассмотрим вертикальный взлёт, парение и посадку, в этом случае угловые скорости всех четырех винтов совпадают и равны $\omega$. Выберем управление таким образом, чтобы $z(t)$ и $\omega(t)$ были гладкими непрерывными функциями. Для их реализации разделим управление на 3 этапа:

1) при $0 \leq t<5 \omega(t)$ меняется по квадратичному закону, происходит набор высоты;

2) при $5 \leq t<10 \omega=g m / 4 k_{1}=37,73$ рад/с парение на высоте $h=50 \mathrm{M}$;

3) при $10 \leq t<15 \omega(t)$ меняется по аналогичному квадратичному закону, происходит посадка.

Отметим, что на интервале $0 \leq t<5$ координата $z(t)$ являлась функцией четвертой степени от $t$ (рис. 2).

Соответствующие графики приведены ниже на рис. 2. 

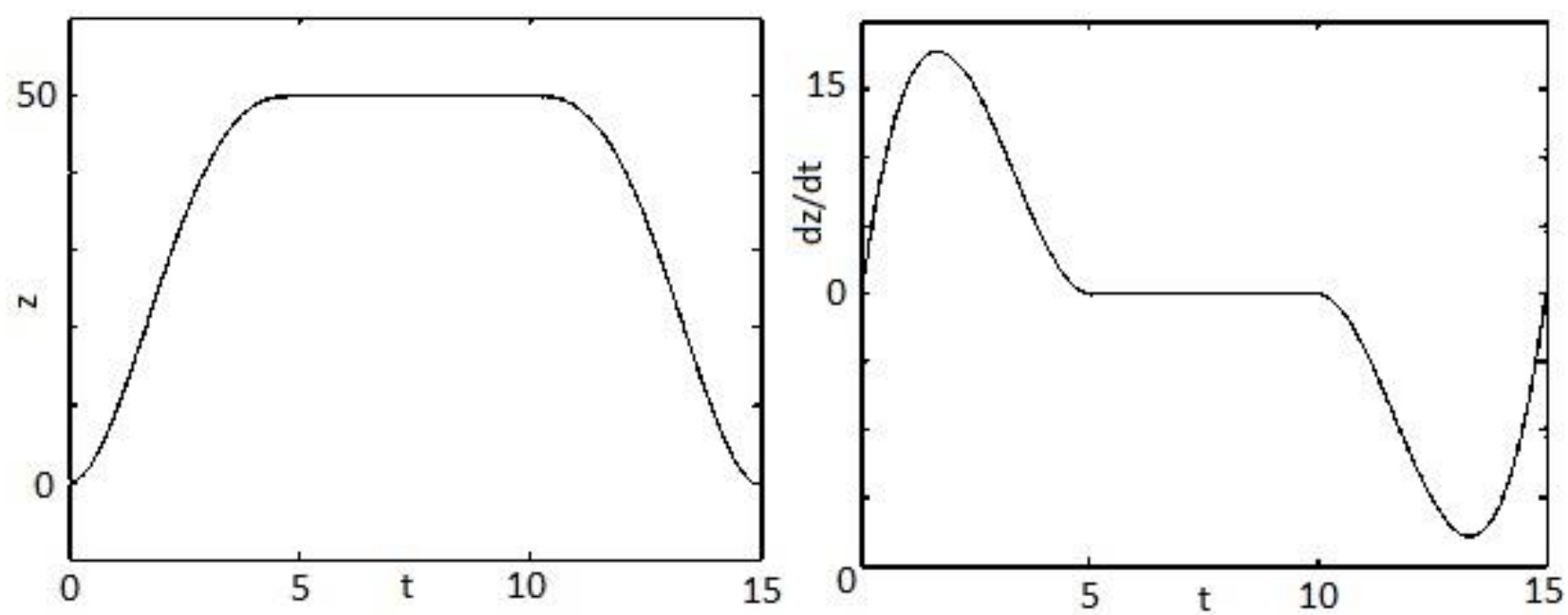

Puc. 2. График зависимости высоты и абсолютной скорости от времени при взлете-парении-посадке

\section{2. Полет по горизонтальной прямой}

Без ограничения общности рассмотрим движение вдоль прямой $x=0, z=0$. Также предположим, что $\varphi \equiv 0$. Тогда из уравнений движения следует, что $\psi \equiv 0$. В результате, получаем, что одним из вариантов осуществления полета по прямой является движение квадрокоптера в плоскости $O z y$ при некотором фиксированном угле крена $\theta_{0}$. В данном случае управления следует выбирать следующим образом $u_{i}=M g /\left(4 k_{1} \cos \theta_{0}\right)$. При этом получаем равноускоренный полет по прямой с ускорением $-\operatorname{gtg} \theta_{0}$.

Также стоит отметить, что управление можно подобрать таким образом, чтобы квадрокоптер осуществлял движение по прямой с остановкой в заданной точке. Для этого необходимо решить обратную задачу динамики, которая сводится к решению одного дифференциального уравнения первого порядка.

\section{3. Нейросетевой метод управления}

В данном разделе исследуем нейросетевой метод синтеза управления. Он, в частности, позволяет преодолеть проблему дефицита управлений.

Суть нейросетевого подхода к управлению летательными аппаратами заключается в создании многоуровневого алгоритма (рис. 3), способного автономно адаптироваться к непрогнозируемым внешним возмущениям и погрешностям измерительных устройств. В самом верхнем контуре происходит формирование целей и ключевых параметров полета. Например, необходимо переместиться из точки А в точку В или следовать за подвижной целью, облетая возникающие на пути препятствия. 
Внешний контур управления

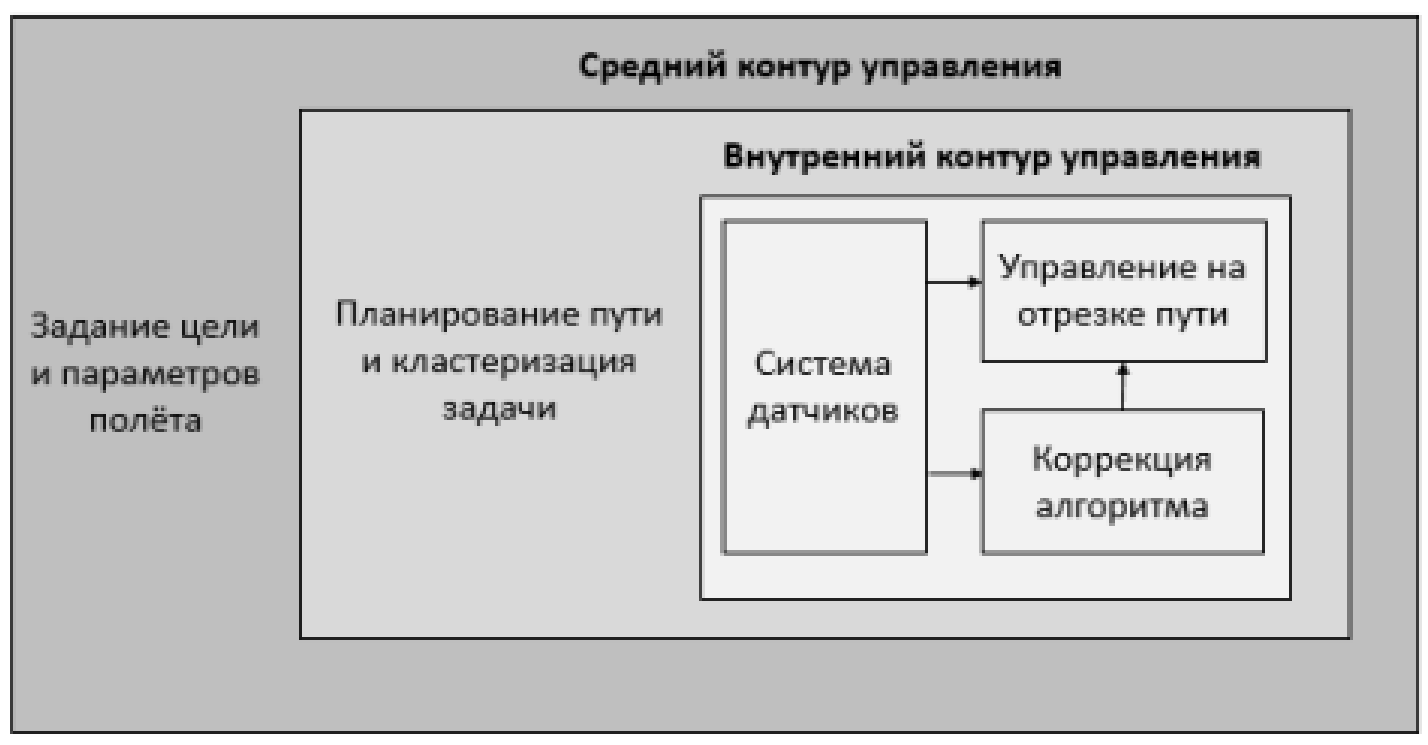

Puc. 3. Контуры алгоритма управления

В следующем контуре происходит интерпретация заданных параметров в функциональные ограничения. Также на нем осуществляется планирование общей траектории полета. Оно состоит из разделения маршрута на отрезки таким образом, что на каждом из них траектория полета в требуемой степени близка к некоторому шаблонному значению. Причем это шаблонное значение задано и описано наперед и реализуется с высокой скоростью. Данный контур можно назвать выделением типовых решений или кластеризацией задачи.

Внутренний контур состоит из нескольких отдельных блоков. Во-первых, это ряд нейросетевых регуляторов, отвечающих за движение по базовым траекториям (шаблонам). Во-вторых, необходим блок, который будет отвечать за коррекцию алгоритма путем обновления обучающей выборки натурными испытаниями. Также можно добавить блок, отвечающий за создание новых шаблонов на основании многократных повторений подобных маневров.

В данной работе описаны отдельный нейросетевой контроллер и принципы его работы на некоторых базовых траекториях. Подтверждение его удовлетворительной работы в этом случае позволит начать разработку многоуровневого контроллера, адаптирующегося к непредсказуемым внешним факторам и способного к самостоятельной корректировке.

Ниже приведена схема синтеза управления с использованием нейросетевого контроллера, состоящая из трех основных функций: блока, моделирующего датчики, нейронной сети и интегратора. 


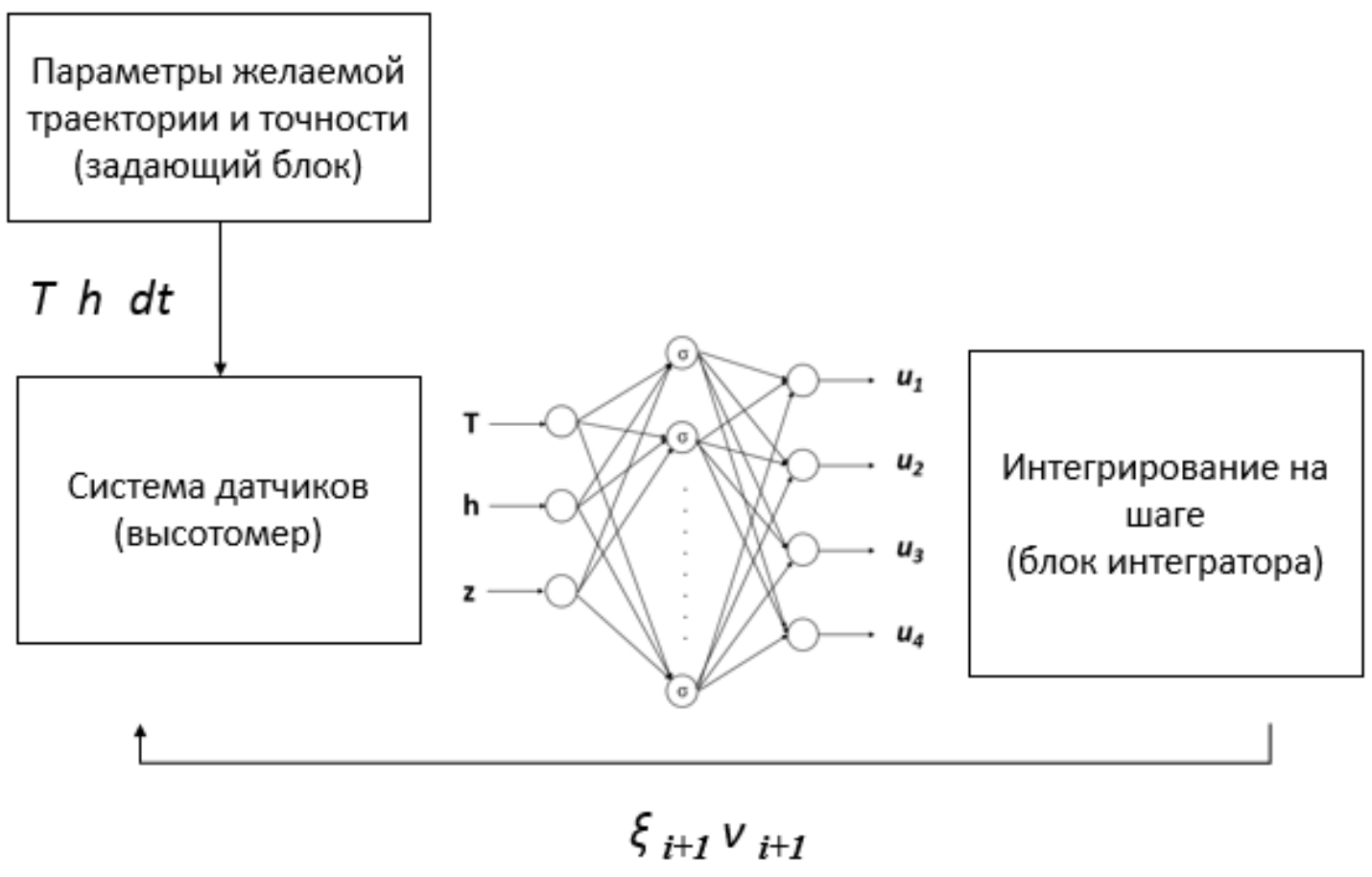

Рис. 4. Вычислительная схема модели

\section{1. Система датчиков}

Наиболее распространенными датчиками являются высотомер, система определения долготы и широты (датчик GPS, система маяк-радар), акселерометры и гироскопы. Для случая вертикального взлета система датчиков состоит из одного гибридного высотомера (предполагается, что для высоты от 0 до 1 м задействован ультразвуковой дальномерный датчик, а для высот более 1 метра подключается барометрический). Он передает сигнал с текущей координатой $z$ в блок нейронной сети с интервалом 0,01 секунды.

\section{2. Блок Нейронной сети (НС)}

Задачей этого блока является вычисление управляющих воздействий с помощью нейронной сети в зависимости от текущих параметров системы и требуемой точности. Он позволяет находить функции управления в задачах с плохо моделируемыми или непредсказуемыми эффектами. Так, например, для корректировки управления при появлении бокового ветра не обязательно знать его силу, а достаточно знать показания бортовых датчиков. Являясь хорошим аппроксиматором, нейронная сеть позволяет осуществлять быстрые вычисления. Единственным ее недостатком является необходимость в вычислении коэффициентов весовой матрицы, т.е. обучении.

В основе данного блока лежит трехслойная нейронная сеть прямого распространения (трехслойный перцептрон). В результате экспериментов была выбрана сеть с сигмоидальными функциями в скрытом слое, состоящем из 10 нейронов (рис. 5). Вычисление весовой матрицы и смещений осуществляется путем супервизорного обучения [15].

Правило супервизорного обучения предполагает набор примеров (обучающая выборка) правильной работы сети: 


$$
\Xi=\left\{\left(\mathrm{a}_{k}, \mathrm{~b}_{k}\right), \mathrm{k}=1, \ldots, N\right\},
$$

где $a_{k}-$ вход в нейронную сеть и $b_{k}-$ соответствующий ему правильный выход (цель). Когда входы предъявляются сети, её выходы сравниваются с целями. Правило обучения используется для уточнения весов и смещений нейронов сети, чтобы приблизить выход сети к соответствующим целям, которые указаны в задающем блоке.

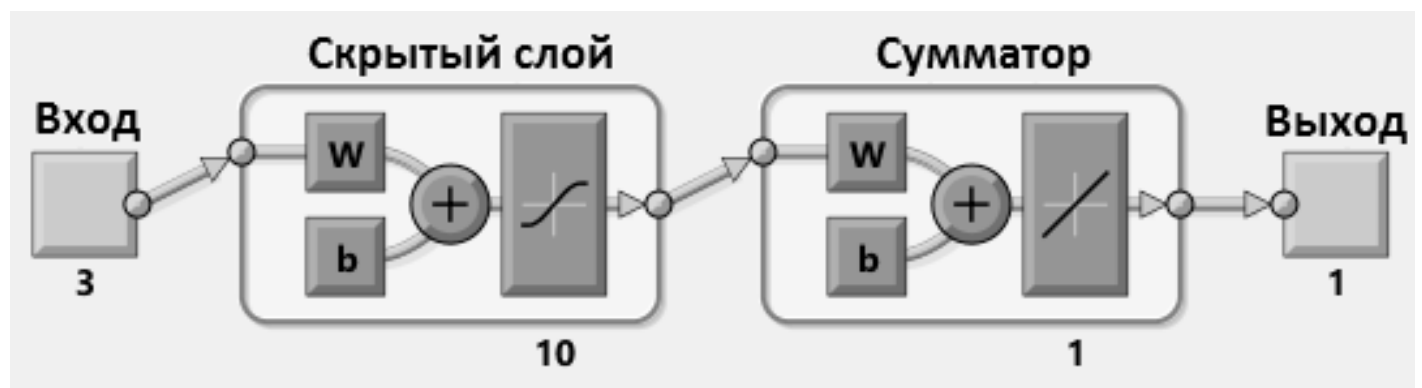

Puc. 5. Схема нейронной сети для взлёта на высоту $h$ за время $T$ (рисунок дан в нотации MATLAB)

Обучающая выборка формируется следующим образом. Для начала составляется семейство функций, которыми будет приближаться целевое решение. Далее с помощью численного моделирования решается обратная задача динамики по вычислению соответствующего семейства функций управления. Полученное семейство формирует набор выходов нейронной сети $b_{k}$. В качестве входов используются параметры целевой траектории (высота и время подъема-парения-посадки, угол поворота и др.), а также информация, поступающая с блока датчиков (текущая координата центра масс, скорость и др.).

Например, для случая вертикального взлета за время $t_{l}$ на высоту $h$ семейством целевых функций выбрано множество многочленов четвертой степени следующего вида:

$$
\tilde{z}_{n}=\frac{3 h}{t_{1}^{4}} t^{4}-\frac{8 h}{t_{1}^{3}} t^{3}+\frac{6 h}{t_{1}^{2}} t^{2}
$$

в котором коэффициенты найдены из условий, что $\tilde{z}_{n}(0)=0, \tilde{z}_{n}\left(\mathrm{t}_{1}\right)=h, \dot{\tilde{z}}_{n}(0)=0, \dot{\tilde{z}}_{n}\left(\mathrm{t}_{1}\right)=0, n-$ количество функций в семействе. Причем каждому такому многочлену соответствует функция управления. Таким образом, для случая вертикального взлета с датчиком высоты (рассмотренного далее при моделировании) обучающей выборкой является множество строк вида:

$$
\left(\mathrm{t}_{1}, h, \tilde{z}_{n}\left(\mathrm{t}_{j}\right), u_{n}\left(\mathrm{t}_{j}\right)\right),
$$

где $j$ - соответствует количеству точек на целевой функции, взятых для обучающей выборки, $\tilde{z}_{n}\left(\mathrm{t}_{j}\right)$ - текущая высота и $u_{n}\left(\mathrm{t}_{j}\right)$ - управляющее воздействие (сила вертикальной тяги). Первые три являются входными параметрами сети, а последнее - выходом. 
Задачей этого блока является вычисление управляющих воздействий с помощью нейронной сети в зависимости от текущих параметров системы и требуемой точности.

\section{3. Блок интегратора}

Данный блок интегрирует систему уравнений Лагранжа с учетом входящих параметров $(T, h, z)$, в том числе управляющих воздействий. На выходе получается новое состояние системы, которое на следующем шаге цикла моделирования опять подается в нейронную сеть и т.д.

\section{4. Вертикальный взлет}

Случай вертикального взлета является одномерной задачей с переменной $z$. Система датчиков состоит из одного гибридного высотомера (предполагается, что для высоты от 0 м до 1 м задействован ультразвуковой дальномерный датчик, а для высот более 1 метра подключается барометрический). Он передает сигнал в блок нейронной сети с интервалом 0,03 секунды.

В качестве параметров желаемой траектории выберем желаемую высоту подъема $(h)$ и время подъема $\left(t_{l}\right)$. Для начала рассмотрим работу предложенного алгоритма для взлета на высоту 3 м и 5 м за 3 с без учета погрешности высотомера (рис. 6).

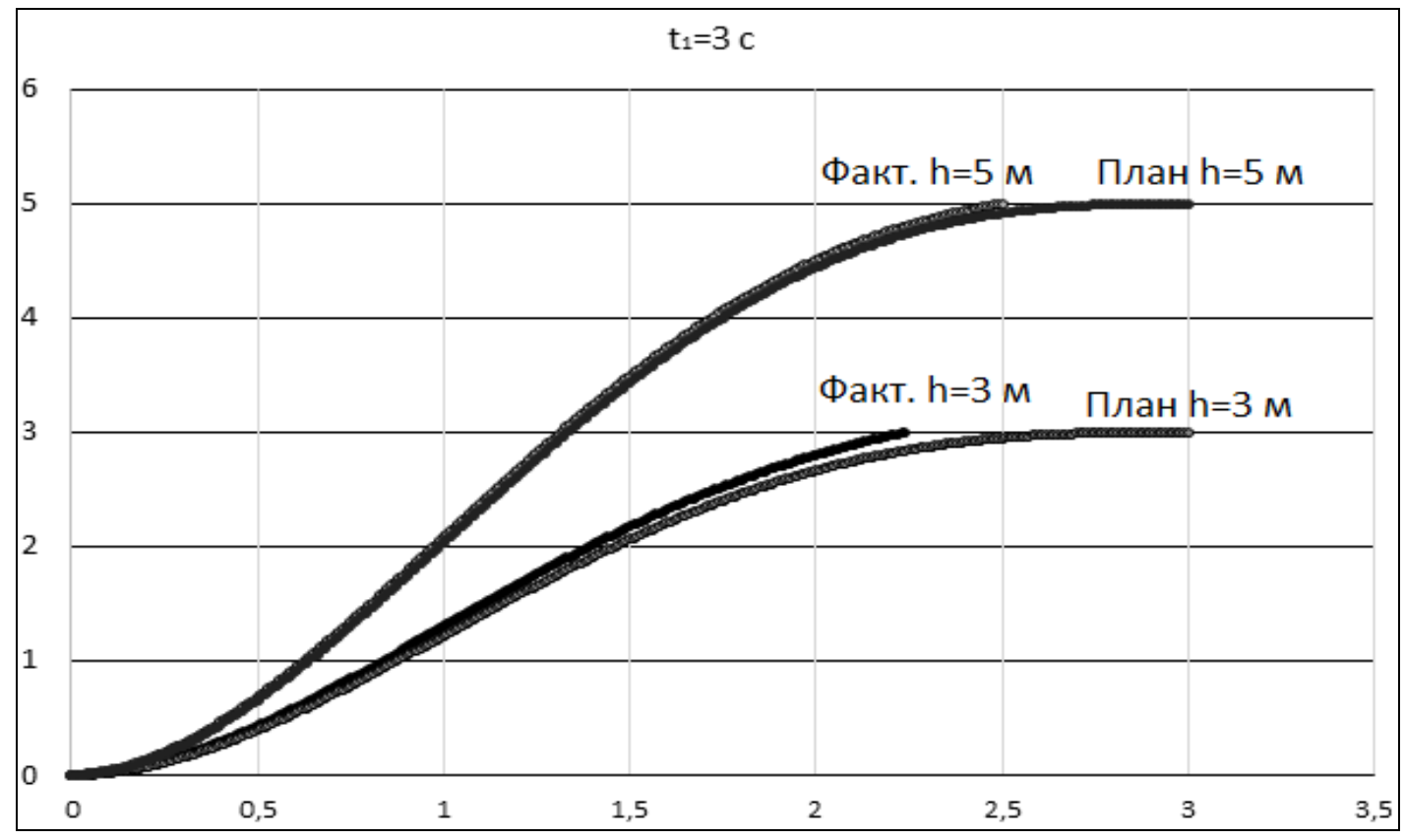

Puc. 6. Взлет на высоту 3 и 5 м с идеальными датчиками высоты

Графики, соответствующие фактической работе регулятора, более краткие по времени в связи с тем, что при достижении целевой высоты алгоритм моделирования останавливается. 


\section{5. Погрешность системы для случая вертикального взлета}

В данной системе были учтены погрешность измерения датчика высоты и наличие шума. Шум и погрешность определялись как некоторые случайные числа, находившиеся в определенном диапазоне. На рис. 7 и 8 показана работа регулятора в зависимости от величины максимальной погрешности на шаге. Для случая с погрешностью 4 см среднеквадратичное отклонение от целевой траектории для взлета на 3 м составило 0,13 , на 5 м - 0,29. Для случая с погрешностью 10 см среднеквадратичное отклонение от целевой траектории для взлета на 3 м составило 0,5 , на 5 м $-0,09$.

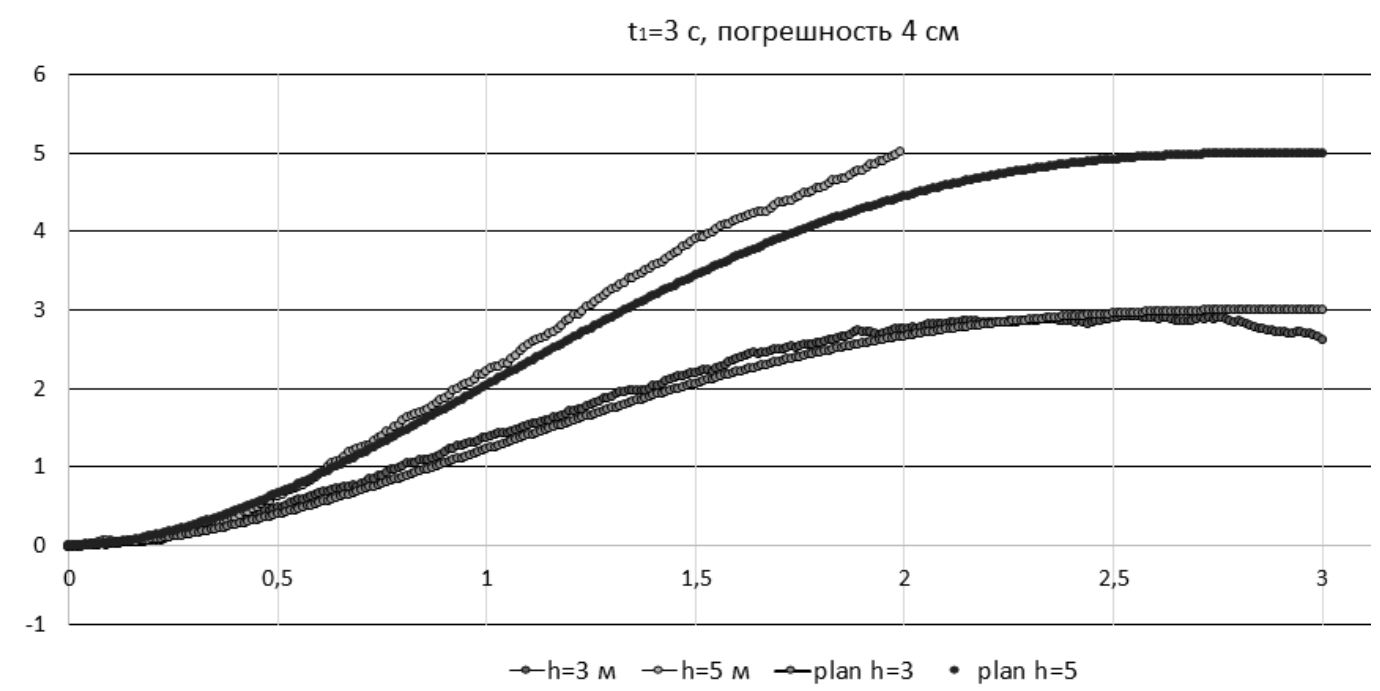

Рuc. 7. Взлет за 3 с на высоту 3 м и 5 м с погрешностью высотомера 4 см

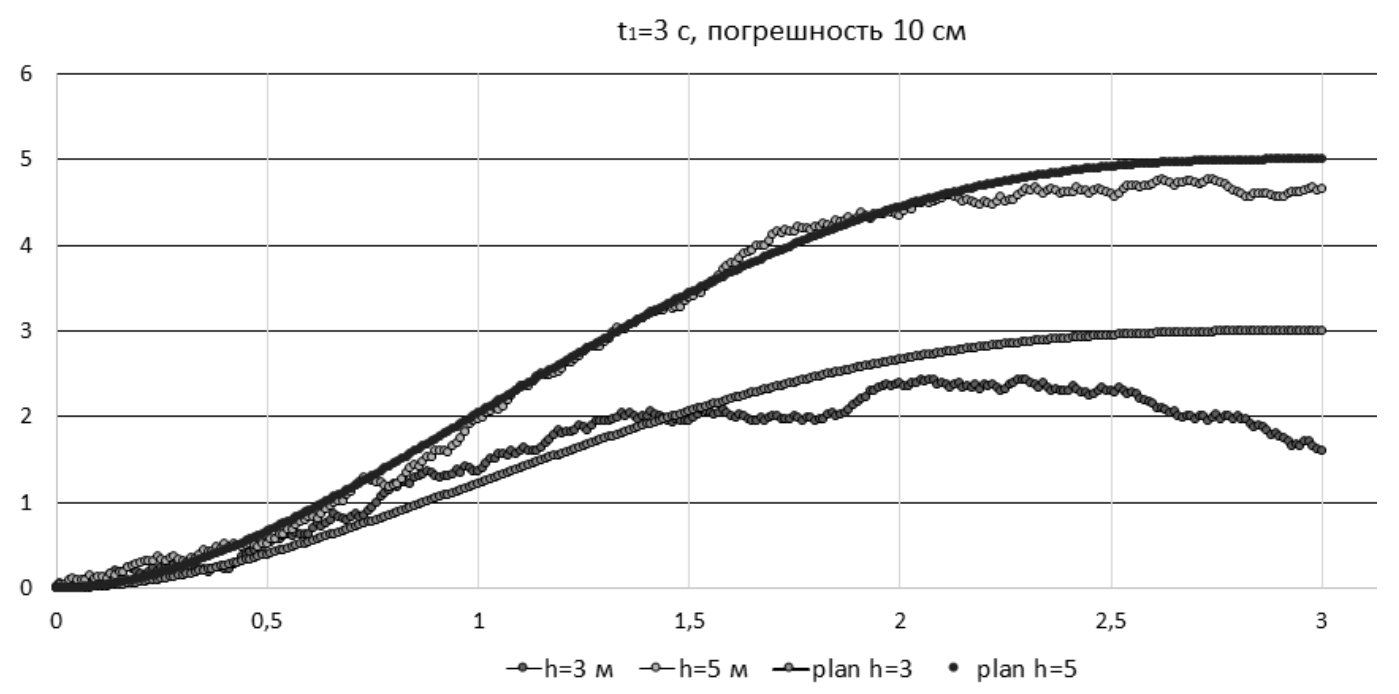

Puc. 8. Взлет за 3 с на высоту 3 м и 5 м с погрешностью высотомера 10 см

На основании серии экспериментов для взлета на заданную высоту за заданное время было получено, что при погрешности датчика высоты до 4 см происходит существенное отклонение траектории от желаемой (не более 5\% от общей длины пути). Проведенное моделирование показало достаточно эффективную работоспособность этой схемы для меньших погрешностей. 
Предложенный в данной работе нейросетевой регулятор удовлетворительно работает для точности высотомера порядка 4 см.

\section{6. Управление углом крена}

Аналогично можно построить нейросетевой контроллер для управления углами. Например, для управления углом крена обучающей выборкой является множество строк вида:

$$
\left(T, \theta_{1}, \theta, u_{1}, u_{2}, u_{3}, u_{4}\right) \text {, }
$$

где $T$ - длительность поворота, $\theta_{1}$ - угол отклонения, $\theta$ - текущий угол крена и $u_{i}$ - управляющие воздействия. В качестве целевого управления выбраносемейство функций вида:

$\theta(t)=\theta_{1} \sin ^{2}(\pi t / 2 T+\pi / 2)$, где $T$ и $\theta_{1}-$ параметры обучающей выборки.

Рассмотрим результаты работы построенного нейросетевого контроллера для $\theta_{1}=\pi / 12, t_{1}=5 \mathrm{c}$. На рис. 9 показаны график целевой функции и результат работы нейросетевого алгоритма для поворота угла крена с $\pi / 12$ до нуля.

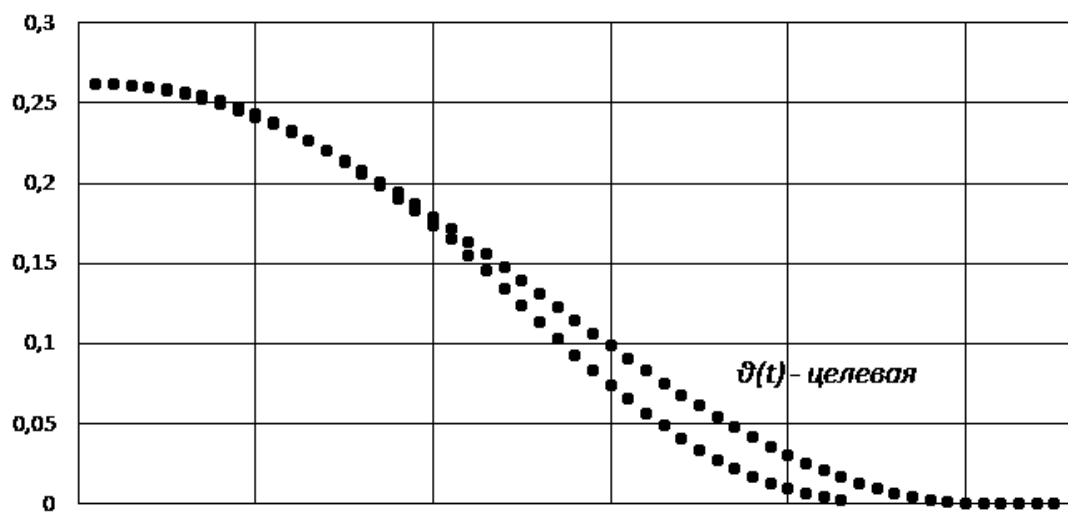

Puc. 9. Поворот на угол $\pi / 12$

График, соответствующий работе регулятора, достаточно хорошо приближает целевую функцию: среднеквадратичное отклонение составляет 0,015. Это позволяет говорить об удовлетворительной работе построенного алгоритма.

\section{5. Заключение}

Построена модель квадрокоптера с четырьмя управлениями и шестью степенями свободы, учитывающая аэродинамические эффекты через воздействие воздуха на вращение лопастей винтов. С помощью численного моделирования рассматривались взлет-парение-посадка и полет по прямой. Рассмотрено нейроуправление коптером, рассмотрены случаи работы регулятора для управления углом поворота, для взлета на заданную высоту с учетом погрешности датчика высоты и без нее. 
В результате проведенного эксперимента по моделированию показана возможность реализации нейроконтроллера управления квадрокоптером на типовых траекториях. Проведен анализ работы алгоритма для серии различных значений погрешности датчиков и случайного шума. Показано, что для рассмотренного конкретного варианта квадрокоптера (характерный размер порядка 1 м) нейрорегулятор взлета-посадки удовлетворительно работает при погрешности датчиков высоты порядка 4 см.

Настоящая работа выполнена при частичной финансовой поддержке гранта РФФИ 15-07-07483 А.

\section{Библиографический список}

1. Avila Vilchis J.C., Brogliato B., Dzulc A., Lozano R. Nonlinear modelling and control of helicopters,. Automatica 39, 2003.

2. Dzul P. A. and Lozano R. Real-time stabilization and tracking of a four-rotor mini rotorcraft. IEEE Transaction on Control System Technology, 12(4):510 - 516, July 2004.

3. Bresciani T. Modelling, Identification and Control of a Quadrotor Helicopter, Department of Automatic Control, Lund University, 2008.

4. Фантони И., Лозано Р. Нелинейное управление механическими системами с дефицитом управляющих воздействий. - Москва-Ижевск: ООО "Компьютерная динамика", 2012.

5. Евгенов А.А. Нейросетевой регулятор системы управления квадрокоптером. ФГБОУ ВПО «Южно-Российский государственный политехнический университет (НПИ) имени М.И. Платова».

6. Madani T., Benallegue A. Adaptive Control via Backstepping Technique and Neural Networks of a Quadrotor Helicopter, Proceedings of the 17th World Congress. The International Federation of Automatic Control, Seoul, Korea, July 6-11, 2008.

7. Hoffmann G.M., Huang H., Waslander S.L., and Tomlin C.J. Quadrotor helicopter flight dynamics and control: Theory and experiment, Proceedings of the AIAA Guidance, Navigation and Control Conference and Exhibit, Aug. 2007, 1-20.

8. Pounds P., Mahony R., Corke P. Modelling and Control of a Quad-Rotor Robot, Canberra, Australian National University, 2008, 1-10.

9. Hana Boudjedir, Fouad Yacef, Dual neural network for adaptive sliding mode control of quadrotor helicopter stabilization // International Journal of Information Sciences and Techniques (IJIST) Vol.2, No.4, July 2012. 
10. Joao P., Mendes B. Assisted Teleoperation of Quadcopters Using Obstacle Avoidance. Lisbon. 2012.

11. Nicol C., Macnab C.J.B., Ramirez-Serrano A. Robust neural network control of a quadrotor helicopter // Mechatronics. Volume 21. Issue 6. September 2011. P.927938.

12. Dierks T., Jagannathan S. Neural Network Control and Wireless Sensor Networkbased Localization of Quadrotor UAV Formations // Aerial Vehicles. 2009. P. 601-620.

13. Munoz R.S.M., Rossi C., Cruz A.B. Modelling and Identification of Flight Dynamics in Mini-Helicopters Using Neural Networks // Aerial Vehicles. 2009. P. 287-312.

14. Lavi B. An Adaptive Neuro PID for Controlling the Altitude of quadcopter Robot // International Conference on Methods and Models in Automation and Robotics. Poland. Volume: 18th. 2014. P.662-665.

15. Голубев Ю.Ф. Нейронные сети в мехатронике // Фундаментальная и прикладная математика. 2005, т.11, № 8, С. 81-103.

16. Павловский В.Е., Савицкий А.В. Исследование обратной задачи для вычисления управляющих воздействий для квадрокоптера. // Препринты ИПМ им. М.В.Келдыша. 2017. № 17. 20 с.

doi:10.20948/prepr-2017-17

http://keldysh.ru/papers/2017/prep2017_17.pdf 


\section{Оглавление}

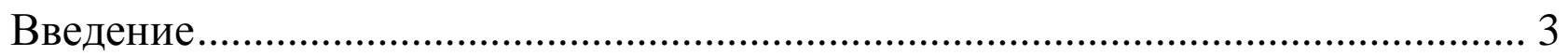

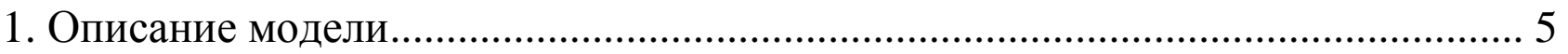

1.1. Основные предположения .................................................................... 5

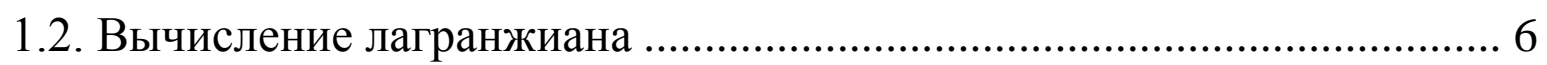

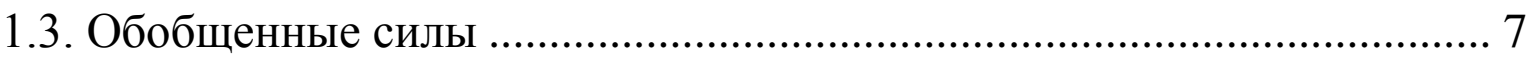

1.4. Модель подъемной силы и момента несущего винта ............................ 8

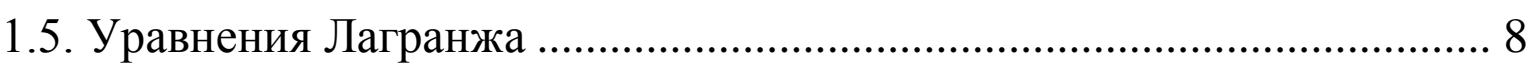

2. Моделирование и алгоритмы управления ………............................................... 10

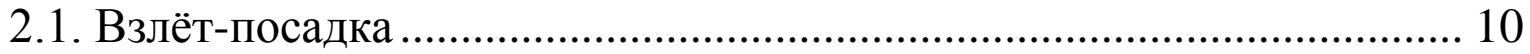

2.2. Полет по горизонтальной прямой ...................................................... 11

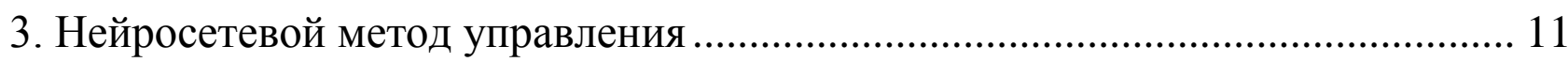

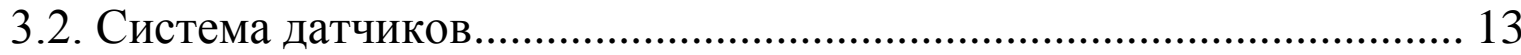

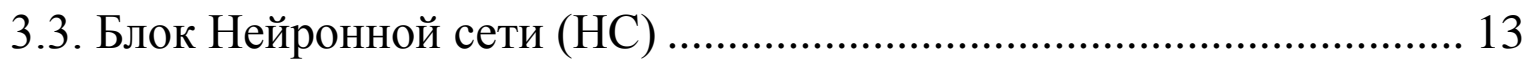

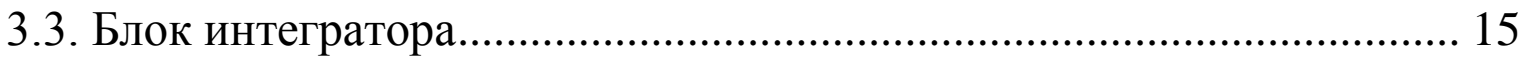

3.4. Вертикальный взлет ....................................................................... 15

3.5. Погрешность системы для случая вертикального взлета ................. 16

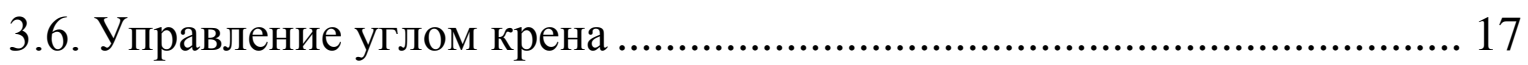

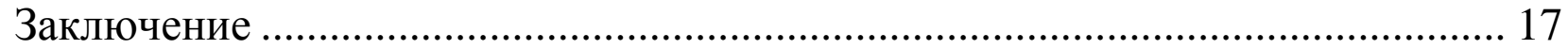

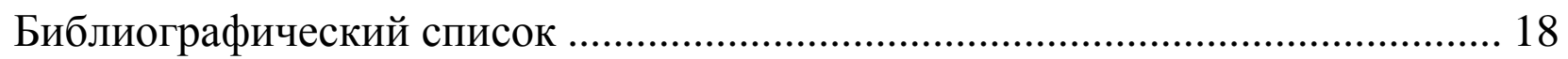

